\title{
DETECTION OF KNOWN FM SIGNALS IN KNOWN HEAVY-TAILED NOISE
}

\author{
B. Barkat, M.Y. Siyal and J. Yingtuo \\ Nanyang Technological University \\ School of EEE, Block S2 \\ Nanyang Avenue, Singapore 639798
}

\begin{abstract}
In this paper, we consider the detection of a known frequency modulated signal corrupted by known heavy-tailed additive noise. Two different techniques will be presented. The first one is based on the Neyman-Pearson theorem and the second technique is based on the time-frequency distribution of the signal. A statistical performance comparison, using the receiver operating characteristics (ROC), of the two methods is also presented.
\end{abstract}

\section{INTRODUCTION}

In many engineering applications such as radar, sonar, telecommunications, the signal is assumed to be corrupted by additive Gaussian noise. This assumption is usually made for mathematical simplicity and may be justified by the central limit theorem. Although the Gaussian assumption yields acceptable solutions for modeling most real-life signals, there are many situations wherein this assumption does not hold. In these cases, other noise models and subsequently alternative analysis techniques are needed.

In this paper, we consider heavy-tailed noise processes which are characterised by infrequent but high level events. This kind of noise, also known as impulsive noise, can be encountered in many engineering applications. For instance, impulsive noise can be due to thunderstorms or by an iceberg breakup in under ice acoustic [1]. Modeling these events by Gaussian models will lead to poor detection performances.

A class of distributions adopted by the signal processing community to model the statistical behavior of impulsive processes is the heavy-tailed distributions class. Examples of heavy-tailed distributions include Laplace, Cauchy and $\alpha$-stable distributions with $\alpha<2$. The use of these distributions has proved to be effective in modeling many real-life engineering problems [2].

In this paper, we propose to address the problem of detecting known non-stationary FM signals corrupted by known additive heavy-tailed noise. Two different methods will be presented here. The first method is based on the Neyman-Pearson method and the second method is based on the time-frequency distribution (TFD) of the signal. We perform a statistical comparison of the two methods and show, using the receiver operating characteristics (ROC) of the detectors, the superiority of the time-frequency based detection method.

The paper is organised as follows. In Section 2 we discuss the Neyman-Pearson based detector. In Section 3, we present the time-frequency based detector and give a performance comparison of the two detectors. Section 4 concludes the paper.

\section{NEYMAN-PERSON BASED DETECTION METHOD}

In this section, we consider the detection of a known signal in independent and identically distributed (i.i.d) impulsive noise with known characteristics. For that, let us consider the following detection problem:

$$
\begin{array}{lll}
\mathcal{H}_{0}: x[n] & =w[n] & n=0,1, \ldots, N-1 \\
\mathcal{H}_{1}: x[n] & =A s[n]+w[n] & n=0,1, \ldots, N-1
\end{array}
$$

where the signal $A s[n]$ (with $A>0$ ) is known and $w[n]$ are i.i.d. noise samples with known probability density function (pdf) $p(w[n])$. The Neyman-Pearson (NP) detector decides $\mathcal{H}_{1}$ if the likelihood ratio exceeds a threshold $\gamma$, i.e.,

$$
L(\mathbf{x})=\frac{p\left(\mathbf{x} ; \mathcal{H}_{1}\right)}{p\left(\mathbf{x} ; \mathcal{H}_{0}\right)}>\gamma
$$

Due to the i.i.d assumption, the above result is equivalent to

$$
L(\mathbf{x})=\frac{\prod_{n=0}^{N-1} p\left(x[n] ; \mathcal{H}_{1}\right)}{\prod_{n=0}^{N-1} p\left(x[n] ; \mathcal{H}_{0}\right)}
$$

It can be shown that the NP detector decides $\mathcal{H}_{1}$ if [1]

$$
\sum_{n=0}^{N-1} g_{n}(x[n])>\gamma^{\prime}
$$

where

$$
g_{n}(x[n])=\ln \frac{p(x[n]-A s[n])}{p(x[n])} .
$$

The determination of the probability of false alarm, $P_{F A}$, and probability of detection, $P_{D}$, for the above detector is difficult due to the nonlinearity in the test statistic $\sum_{n=0}^{N-1} g_{n}(x[n])$ [1]. That is, the above detector can not be implemented because the corresponding threshold cannot be determined. To avoid this problem, an asymptotic analysis method has been proposed in [1]. This method, which yields an equivalent asymptotic detector, examines the NP detector as $A \rightarrow 0$ (i.e., weak signals). The quantity $g_{n}(x[n])$ is viewed as a function of $A$ and expanded using a first-order Taylor series about $A=0$. Doing so produces

$$
\begin{aligned}
g_{n}(x[n]) & =\ln \frac{p(x[n]-A s[n])}{p(x[n])} \\
& \approx 0+\left.\frac{\frac{d p(w)}{d w}}{p(w)}\right|_{w=x-A s[n], A=0}(-s[n] A)
\end{aligned}
$$




$$
\approx-\frac{\frac{d p(x)}{d x}}{p(x)} s[n] A
$$

Thus, the asymptotic detector decides $\mathcal{H}_{1}$ if

$$
\sum_{n=0}^{N-1} g_{n}(x[n]) \approx \sum_{n=0}^{N-1}-\frac{\frac{d p(x)}{d x}}{p(x)} s[n] A>\gamma^{\prime}
$$

or equivalently,

$$
T(\mathbf{x})=\sum_{n=0}^{N-1}-\frac{\frac{d p(x)}{d x}}{p(x)} s[n]>\gamma^{\prime \prime} .
$$

The above weak signal NP based detector, which can be shown to be locally optimum [1], is displayed in the Block diagram below. This detector consists of the nonlinearity function $g(x)=$ $-(d p(x) / d x) / p(x)$ followed by a replica-correlator. The nonlinearity acts to limit the samples that are large in magnitude. That is, the NP based detector attempts to reduce the effect of the noise outliers.

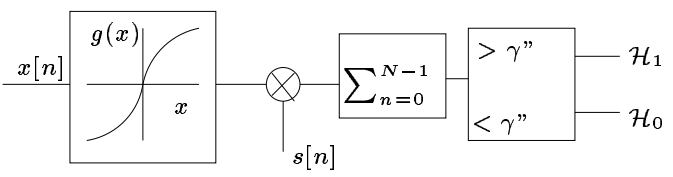

Block diagram of the NP based detector

Note that for the Gaussian noise it can easily be shown that $g(x)=x / \sigma^{2}$ and in this case the test statistic $T(\mathbf{x})$ reduces to

$$
T(\mathbf{x})=\frac{1}{\sigma^{2}} \sum_{n=0}^{N-1} x[n] s[n]
$$

which is the well known replica correlator.

In order to evaluate the performance of the above asymptotic detector we need the pdf of the test statistic $T(\mathbf{x})$ under both hypothesis $\mathcal{H}_{0}$ and $\mathcal{H}_{1}$. Applying the central limit theorem, these can be shown to be asymptotically given by [1]

$$
T(\mathbf{x}) \stackrel{a}{\sim}\left\{\begin{array}{l}
\mathcal{N}\left(0, i(A) \sum_{n=0}^{N-1} s^{2}[n]\right) \quad \text { under } \mathcal{H}_{0} \\
\mathcal{N}\left(A i(A) \sum_{n=0}^{N-1} s^{2}[n], i(A) \sum_{n=0}^{N-1} s^{2}[n]\right) \\
\quad \text { under } \mathcal{H}_{1}
\end{array}\right.
$$

where

$$
i(A)=\int_{-\infty}^{\infty} \frac{\left(\frac{d p(w)}{d w}\right)^{2}}{p(w)} d w
$$

is called the Fisher information or the intrinsic accuracy of the pdf $p(w)$. We see that the test statistic $T(\mathbf{x})$ has the performance of the NP detector for the mean-shifted Gauss-Gauss detection problem and, consequently, it can be shown that [1]

$$
P_{D}=Q\left(Q^{-1}\left(P_{F A}\right)-\sqrt{d^{2}}\right)
$$

where $d^{2}$ is the deflection coefficient given by

$$
\begin{aligned}
d^{2} & =\frac{\left(E\left(T(\mathbf{x}) ; \mathcal{H}_{1}\right)-E\left(T(\mathbf{x}) ; \mathcal{H}_{0}\right)\right)^{2}}{\operatorname{var}\left(T(\mathbf{x}) ; \mathcal{H}_{0}\right)} \\
& =A^{2} i(A) \sum_{n=0}^{N-1} s^{2}[n]
\end{aligned}
$$

and $Q(x)$ is the right-tail probability or the complementary cumulative distribution given by

$$
Q(x)=\int_{x}^{\infty} \frac{1}{\sqrt{2 \pi}} \exp \left(-\frac{1}{2} t^{2}\right) d t
$$

From the above results, we note that the effect of the noise pdf on the asymptotic detection performance is only via $i(A)$. It is also interesting to note that the pdf that yields the smallest $i(A)$, and hence the poorest detection performance, is the Gaussian pdf [1].

Next, we propose a different detector and we compare its performance to the asymptotic detector presented above.

\section{TIME-FREQUENCY BASED DETECTOR}

In this section, we present a time-frequency based detector for known signals corrupted by heavy-tailed noise. To do so, we start by giving a brief review of the time-frequency analysis concept.

\subsection{Time-Frequency Analysis}

The use of the joint time-frequency representation gives us a better understanding in the analysis of non-stationary signals, i.e. signals whose spectral contents are time-varying. The ability of the TFD to display the components temporal localisation of a given nonstationary signal makes it a very powerful tool in the localisation and estimation of the instantaneous frequency (IF) of the signal [3, 4]. To illustrate this point, let us consider the analysis of a linear FM signal.

The signal spectrum, displayed in Figure 1 (bottom plot), gives no indication on how the frequency of the signal is changing with time. The time domain representation (same figure left plot) is also limited and does not provide full information about the signal. A joint time-frequency representation of the signal is displayed in the right plot of Figure 1. It is clear from this figure that one can easily notice the linear relation between the frequency and the time. Moreover, the analyst can also identify the start and the stop times of the signal frequency.
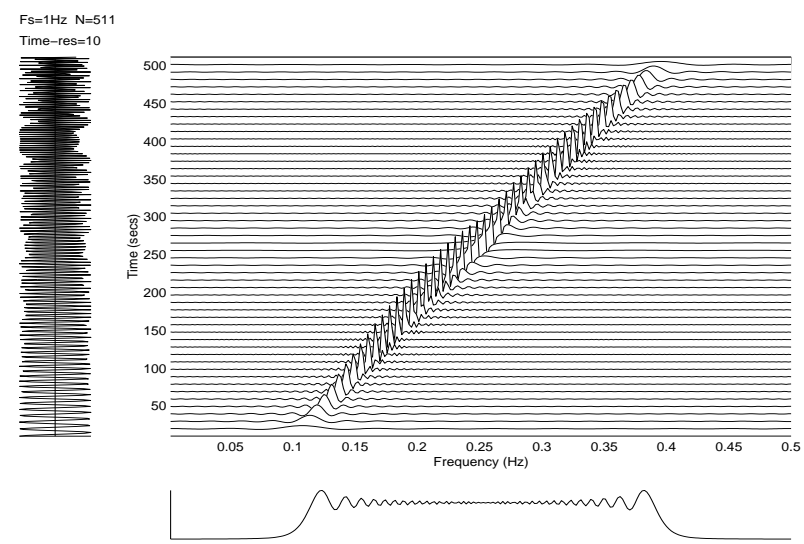

Fig. 1. TFD of a linear FM signal. The signal's time domain representation appears on the left, and its spectrum on the bottom

One of the well known TFDs is the Wigner-Ville distribution 
(WVD) given by [3]

$$
W(t, f)=\int_{-\infty}^{+\infty} z\left(t+\frac{\tau}{2}\right) \cdot z^{*}\left(t-\frac{\tau}{2}\right) e^{-j 2 \pi f \tau} d \tau
$$

where $z(t)$ is the analytic signal associated with the real one. The WVD yields maximum energy concentration about the IF for a linear FM signal. However, this property is not valid for higher-order polynomial, or arbitrary non-linear, FM signals. For such signals, it is advantageous to use the polynomial WVD (PWVD) [5].

\section{2. time-frequency based detector}

Analysis of non-stationary signals affected by additive Gaussian noise has been addressed in details in several places $[6,7,5]$. Here, we study the detection problem when the additive noise is not Gaussian but impulsive.

Since in an impulsive noise the probability of high amplitude noise events is small but their effect on the overall signal-to-noise ratio (SNR) can be quite high, then, we propose to pass the noisy signal through a nonlinear device that compresses the large amplitudes before any further analysis. The output of the nonlinear device (compressor), $x_{\operatorname{comp}}(t)$, may be expressed as

$$
x_{\operatorname{comp}}(t)=|x(t)|^{\beta} \cdot \operatorname{sgn}[x(t)]
$$

where $0<\beta \leq 1$ is a real coefficient that controls the amount of compression applied to the input noisy signal $x(t)$.

If the amplitude of the noiseless signal is very small compared to the impulsive noise spikes, we can obtain at the output of the nonlinear device a big reduction in the variance (i.e., SNR improvement). This improvement yields a better performance in the signal IF estimation using time-frequency distributions. In Figure 2 (top plot), we display the WVD of a non compressed linear FM signal affected by impulsive noise. We observe that the TFD, in this case, does not reveal the real features of the signal. However, by first passing the noisy signal through the compressor and then applying the WVD, we can drastically improve the timefrequency representation. The WVD of the signal (after compression) is displayed in Figure 2 (bottom plot) for $\beta=0.01$.
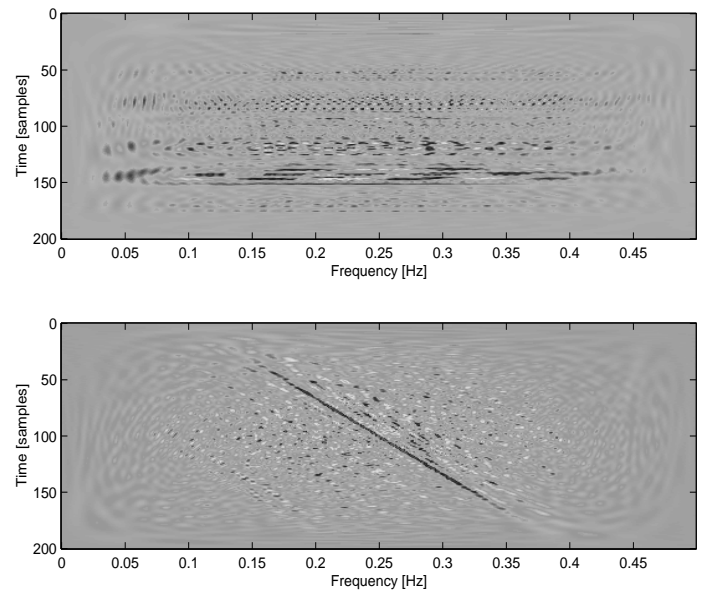

Fig. 2. WVD of a non compressed noisy linear FM signal (top) and the WVDs of the compressed signal for $\beta=0.01$ (bottom).
In order to detect the signal, we need to observe that if a signal is present in the received data, we will have peaks around its IF. If the received data is noise only, we wouldn't have a continuum of peaks around the IF but some peaks randomly distributed in the time-frequency plane. To illustrate this point, let us take slices of the WVDs, at the middle of the signal interval, of a compressed data consisting of a linear FM in impulsive noise and that of a compressed data consisting of noise only. The results of this analysis are displayed in Figure 3. For comparison purposes, we display in the same figure a slice of the WVD, at the same time instant, of the compressed noise-free linear FM signal (dotted curves).
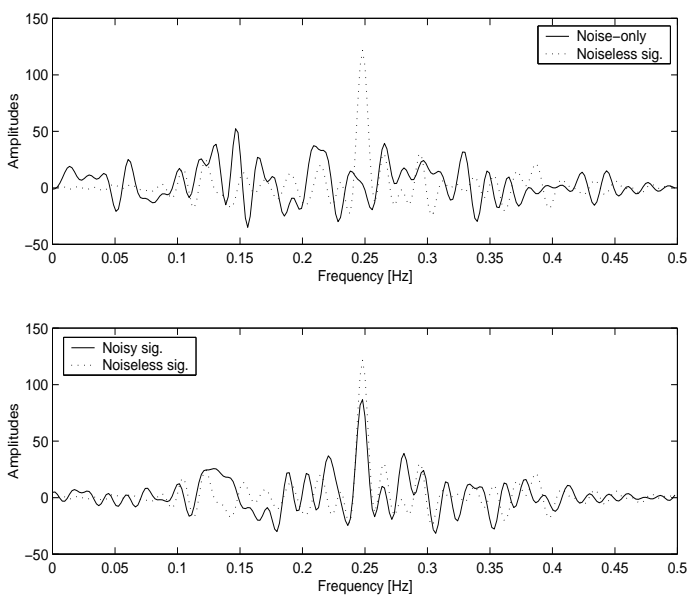

Fig. 3. Slices of the WVDs of a compressed noise-only data (top) and that of a linear FM signal in impulsive noise (bottom). The dotted curve is for the noise-free linear FM signal.

From the above observation, we decide that a signal exists in the received data if the sum of all peaks taken at the signal IF is larger than a fixed threshold. The signal IF is easily estimated from the TFD of the known noise-free signal. The threshold, also obtained from the TFD of the known noise-free signal, is fixed as a percentage of the sum of all peaks taken at the signal IF. The choice of a value of the threshold will yield a particular pair of probability of false alarm and probability of detection. In other words, given the ROC of the detector we can choose any value of the threshold to get a particular performance. Later, we will show how to obtain the ROC for a given situation. The proposed detection algorithm is stated in Table 1.

\subsection{ROC curves and Comparison}

In this subsection, we compare the performance of the proposed time-frequency based detector to the NP based detector using the ROC curves.

We should stress that any monocomponent non-stationary signal can be considered here. However, we limit our discussion to a linear FM signal and we use the WVD as the TFD. Also, we assume that the impulsive noise can be modeled by the standard Cauchy distribution whose pdf is [8]

$$
p(w)=\frac{1 / \pi}{1+w^{2}} .
$$

To obtain the ROC, we run the following experiment. We generate a unit amplitude linear FM signal of length $N=71$ sam- 
1- Pass the noise-free signal through the compressor for a fixed value of $\beta$.

2- Evaluate the TFD of the compressed noise-free signal and get its maximum for every time instant. The signal IF is obtained as the argument of these peaks.

3- Fix the value of the threshold from the sum of these peak values.

4- Pass the received data through the compressor for the same value of $\beta$ chosen in Step 1.

5- Evaluate the TFD of the compressed noisy data and get its values around the signal IF (obtained in Step 2). Sum all these values.

6- Declare a signal present if the sum in Step 5 is larger than the fixed threshold. Otherwise, declare noise only.

Table I. Time-frequency based detection algorithm

ples and a unit sampling period. We evaluate the WVD of the compressed signal, obtain its IF, and choose a threshold value as explained previously. Now, we add impulsive noise to the signal. We evaluate the values of the WVD, of the compressed noisy signal, at the signal IF and sum them. If this sum is larger than the chosen threshold, we declare a signal and increment the detection counter. We do exactly the same for noise only data. For the noise only case, if the sum is larger than the threshold, we declare a false alarm and increment its corresponding counter. For every threshold value, we repeat the experiment 50,000 times. This will give us the $P_{D}$ and the $P_{F A}$ for that particular threshold value. We choose a new value of the threshold and repeat again the whole procedure. We run this experiment for several values of the threshold. At the end, we obtain the ROC by plotting the $P_{D}$ as a function of the $P_{F A}$. The results of the experiment are displayed in Figure 4 for $\beta=0.1$ ('x' and dotted curve). We re-run the same experiment again for $\beta=0.01$ ('x' and continuous curve). The latter gives better results because the high noise spikes have been reduced more through the compressor (i.e., better SNR). This experiment can also be run for other noise pdfs.

For comparison purposes, we display in the same figure the ROC of the NP based detector. For that, we used Equation 2 where $i(A)=0.5$ for the standard Cauchy [8]. We see that the timefrequency based detector is superior to the NP based detector if the $P_{F A}$ is kept lower than 0.25 , which is usually the case in reallife problems.

The proposed detection can be extended to the case of multicomponent FM signals by using a cross-terms free TFD. The peaks of the TFD yields the threshold and the IFs of the components which are, then, used in the TFD of the noisy signal in order to decide the presence of the noise.

\section{CONCLUSION}

In this paper, we consider the detection of a known FM signal corrupted by known impulsive additive noise. We presented a Neyman-Pearson based detector and a time-frequency based one.

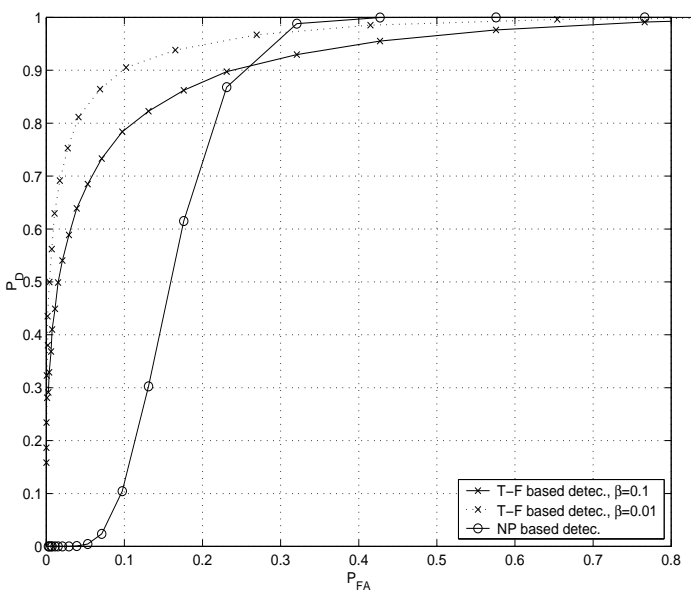

Fig. 4. ROCs for the time-frequency based detector ' $x$ ' and that of the NP based detector 'o'.

A performance comparison of the two detectors, based on their respective receiver operating characteristics, has shown the superiority of the time-frequency based detector for small values of the $P_{F A}$.

\section{REFERENCES}

[1] S. Kay. Fundamentals of Statistical Signal Processing: Detection Theory. Prentice-Hall, 1998.

[2] V. Katkovnik. Robust M-periodogram. IEEE Trans. on Signal Processing, 46(11):3104-3109, Nov. 1998.

[3] L. Cohen. Time-Frequency Analysis. Prentice-Hall, 1995.

[4] F. Hlawatsch and G. F Boudreaux-Bartels. Linear and quadratic time-frequency signal representations. IEEE Signal Processing Magazine, 9(2):21-67, Apr 1991.

[5] B. Barkat and B. Boashash. Instantaneous Frequency Estimation of Polynomial FM Signals Using the Peak of the PWVD: Statistical Performance in the Presence of Additive Gaussian Noise. IEEE Trans. on Signal Processing, 47(9):2480-2490, September 1999.

[6] M. Amin. Time-Frequency Signal Analysis: Methods and Applications, chapter Time-Frequency Spectrum Analysis and Estimation for Non-Stationary Random Processes. LongmanChesire, 1992.

[7] V. Katkovnik and LJ. Stanković. IF estimation using the Wigner distribution with varying and data-driven window length. IEEE Trans. on Signal Processing, Sept. 1998.

[8] Saleem A. Kassam. Signal Detection in Non-Gaussian Noise. Springer-Verlag, 1987. 